\title{
Advanced Carcinoid Tumor
}

National Cancer Institute

\section{Source}

National Cancer Institute. Advanced Carcinoid Tumor. NCI Thesaurus. Code C156487.

A carcinoid tumor that has spread extensively to other anatomic sites or is no longer responding to treatment. 